\title{
Stochastic Models for the Infectivity Function in an Infinite Population of Susceptible Individuals
}

\author{
Viswanathan Arunachalam and Liliana Blanco \\ Department of Statistics, Universidad Nacional de Colombia, Bogotá, Colombia \\ Correspondence should be addressed to Viswanathan Arunachalam; varunachalam@unal.edu.co
}

Received 11 July 2016; Revised 19 December 2016; Accepted 25 December 2016; Published 11 January 2017

Academic Editor: Chin-Shang Li

Copyright (c) 2017 V. Arunachalam and L. Blanco. This is an open access article distributed under the Creative Commons Attribution License, which permits unrestricted use, distribution, and reproduction in any medium, provided the original work is properly cited.

\begin{abstract}
Two stochastic models to study the course of the transient behaviour of the total infectivity present in an infinite population of susceptible individuals are developed. The conditional intensity function of the contagion comprises two components: one is due to the external sources only and the other is the contribution of each of the infected persons which is nonstationary in nature. The statistical characteristics of the number of infected individuals at any time are explicitly obtained. Estimation of the model parameters is also indicated.
\end{abstract}

\section{Introduction}

Infectious diseases impact public health and consequently a nation's progress in several ways. Diseases such as influenza, gastroenteritis among humans and bird flu, and foot and mouth diseases among animals are a matter of great public concern. In the face of such emerging diseases, time is of the essence in developing and implementing interventions. The ability to predict the transient infectivity function (infected population) imposed by such epidemics is essential for intervention design. Stochastic modeling of such infectious diseases is the only effective tool available for the health planners for optimal intervention design and their implementation.

From the time Kermack and McKendrick [1] published their classic in the proceedings of the Royal Society, there have been various approaches to describe the dynamics of the spread of the infectious diseases. They include stochastic compartment models, conditional intensity models $[2$, 3], simulation models, inference models [4], Monte Carlo methods [5], and SIR model [6-9]. Recently, Lie algebra approach was used to study susceptible-infected-susceptible (SIS) epidemic models [10, 11].
Kermack and McKendrick [1] model is characterised by the deterministic equation:

$$
S^{\prime}(t)=S(t)\left[\int_{0}^{t} S^{\prime}(t-\tau) g(\tau) d \tau-f(t)\right]
$$

where $S(t)$ represents the number of susceptible individuals at time $t$. This model is completely characterized by $S(0)$ and the functions $f(t)$ and $g(t)$. The function $f(t)$ models a possible time dependent outside source of infection or represents the history of the epidemic up to time zero. The function $g(t)$ describes the infectivity of an individual as a function of his age of the infection $t$, that is, the time elapsed since the moment of his own infection.

Gielen [12] proposed a natural stochastic version of the above model. He observed that his model fills the gap between Bailey's simple stochastic epidemic model [13] (which is obtained as a special case of Kermack and McKendrik's model when the infectivity parameters $f(t)$ and $g(t)$ are constants) and the Reed-Frost model [12] (when the functions $f(t)$ and $g(t)$ are Dirac delta functions). Gielen [14] further proposed an interesting variant of the above model for a small epidemic in a large population of susceptible individuals. This model 
can be seen to be the direct stochastic version of the Keyfitz [15] deterministic model, governed by the renewal equation:

$$
I^{\prime}(t)=\int_{0}^{t} I^{\prime}(t-\tau) g(\tau) d \tau+f(t),
$$

where $I(t)$ indicates the number of infective individuals at time $t$ in an infinite population of susceptible individuals. Gielen [14] explicitly derived the probability distribution of the number of infective individuals at any time $t$, using which he arrives at several interesting results. However, the expression for the distribution of the number of infective individuals which involves $n$-fold integrals can at best be thought of as a formal solution and is not useful for explicit evaluations. Consequently, the statistical characteristics of the number distribution which are very important in the optimal decision making problems cannot be arrived at that in the general case. One of the purposes of the present work is to analyse the stochastic version of the Keyfitz model [15] and obtain explicitly the statistical characteristics of the number of infective individuals in an infinite population.

The traditional epidemic models assumed the contagion rate to depend on the number of infected persons in the population, thereby tacitly assuming that all individuals exhibit the same pattern of infectiousness. However, this being not so in the reality led to the notion of infectivity function. The infectivity function $f(t)$ is the force of infection at any time $t$ which is the probability/unit of time that a susceptible becomes infected. In the dynamic contagion models, the infectivity functions impact endogenous and exogenous factors of the underlying infection. The proposed stochastic infection models in the present paper split the conditional intensity function of infection into endemic and nonstationary epidemic components. The latter, in our models, is the spread of the disease by person to person contact and provides a description preserve at time $t$ caused by each of the infected individuals. The endemic component models imported cases and is contrary to the epidemic component. The number of infected persons is modeled as a point process with its intensity function dependent on the point process itself, thus providing a more effective model to capture the contagion phenomena. The mean number of infected persons obtained from our stochastic epidemic model, for the special case when both the endemic and epidemic components of the intensity function are constants, satisfies Keyfitz's renewal equation (2). Gielen's stochastic version of Keyfitz's model predicts a negative binomial distribution for the number of infected persons (see [14]) with the same mean thus validating our approach. The proposed formulation is broad enough to handle a wide variety of disease progression models for which infectivity varies both within and external to factors, including Ebola virus disease (EVD) which has generated a large epidemic in West Africa and other parts of the world $[16,17]$.

The present paper is organised as follows. In the next section we propose two stochastic models: the first one is an endemic model in which each person infected from outside source contributes a random amount of infectivity to the force of infectivity (or infectivity function) which decreases at a constant rate over the time. The total infectivity present at any time $t$ is the quantity of interest whose statistical characteristics are obtained. The second model which captures an epidemic has both endemic and epidemic components in the intensity function. The statistical characteristics of the number of infected persons are explicitly obtained. Section 3 presents a discussion on the developed models along with estimation of maximum likelihood parameters of the model.

\section{The Models}

In this section we propose an endemic and an epidemic stochastic model to describe the dynamics of a disease in a large population of susceptible individuals.

2.1. An Endemic Model. We assume that at the time of origin, there are infinitely many susceptible individuals. A susceptible may be infected at time $t>0$, after which it is classified as infected. It is assumed that the random variable denoting the time between two successive infections is exponentially distributed and that infections are due to a source of infection from outside of the population. Our interest is analysing the statistical characteristics of the total force of infectivity $f(t)$ present in the population at any time $t$ due to all the infections until time $t$. In this regard, the contribution to $f(t)$ due to an infection at time $t_{i}(<$ $t$ ) is composed of two components, a discrete jump $a\left(t_{i}\right)$ and a nonstationary component $h\left(t-t_{i}\right)$. Thus the force of infectivity at any time $t$ can be written as follows:

$$
f(t)=\sum_{i=1}^{N_{t}} a\left(t_{i}\right) h\left(t-t_{i}\right),
$$

where $N_{t}$ is the number of infections until time $t$.

From our assumptions, $N_{t}$ is easily seen to be Poisson process. In order to derive the probability frequency functions of the Markov process $f(t)$ we define

$$
\pi(y, t) d y=P(y<f(t)<y+d y \mid f(0)=0) .
$$

The Chapman-Kolmogorov backward equation yields

$$
\begin{aligned}
\pi(y, t)= & (1-\lambda \Delta) \pi(y, t-\Delta) \\
& +\lambda \Delta \pi(y-a h(t), t-\Delta)+o(\Delta),
\end{aligned}
$$

where we have assumed $a\left(t_{i}\right)=a$.

The above equation after simplification yields

$$
\frac{\partial \pi(y, t)}{\partial t}=-\lambda \pi(y, t)+\lambda \pi(y-a h(t), t)
$$

with initial condition

$$
\pi(0, t)=1
$$

Applying the Fourier transform

$$
\tilde{\pi}(s, t)=\frac{1}{2 \pi} \int_{-\infty}^{\infty} \pi(y, t) e^{i s y} d y
$$


to both sides of (6), we obtain

$$
\begin{aligned}
\frac{\partial \widetilde{\pi}(s, t)}{\partial t} & =-\lambda \tilde{\pi}(s, t)+\lambda e^{i s a h(t)} \tilde{\pi}(s, t) \\
& =-\lambda\left(1-e^{i s a h(t)}\right) \tilde{\pi}(s, t) .
\end{aligned}
$$

The above partial differential equation with (7) can be solved to give the solution:

$$
\tilde{\pi}(s, t)=\exp \left(-\lambda t+\lambda \int_{0}^{t} e^{i s a h(u)} d u\right)
$$

Now $\pi(y, t)$ could easily be obtained by inverting (6) for specific forms of the function $h(\cdot)$. In the foregoing model, we have assumed that the contribution of each infected individual to the force of infectivity $f(t)$ is the same constant $a$. However in practice, this may not be so. Thus if we assume the jumps " $a$ " to be a random variable with probability density $p(a)$, then $\pi(y, t)$ in this case can be obtained with minor modifications to the above model. The backward equation (3) now reduces to

$$
\frac{\partial \pi}{\partial t}=-\lambda \pi+\lambda \int_{a} \pi(y-a h(t), t) p(a) d a
$$

and the corresponding equation after applying Fourier transform on both sides turns out to be

$$
\frac{\partial \tilde{\pi}}{\partial t}=-\lambda \widetilde{\pi}+\lambda \int_{a} e^{i s a h(t)} \widetilde{\pi}(s, t) p(a) d a .
$$

Now letting

$$
\phi(u)=\int_{a} e^{i a u} p(a) d a
$$

which is nothing but the characteristic function of the random variable $a$ and using (13) in (12) we obtain

$$
\frac{\partial \tilde{\pi}}{\partial t}=-\lambda \tilde{\pi}+\lambda \tilde{\pi} \phi(\operatorname{sh}(t))
$$

Thus we obtain

$$
\tilde{\pi}=\exp \left(-\lambda t+\lambda \int_{0}^{t} \phi(\operatorname{sh}(\tau)) d \tau\right) .
$$

$\pi(y, t)$ can be obtained by inverting (15) for specific forms of the function $h(t)$.

2.2. An Epidemic Model. In the earlier endemic model, it was assumed that the spread of the infection is due to external source only while each infection contributed a nonstationary input to the force of the infection. The present section develops an epidemic model which spreads due to an external source as well as through contagion of the infected population. Instead of the number of infected persons, we use the more realistic concept of the force of infection. Thus the number of infected at any time $t$ is a point process whose conditional intensity function comprises both endemic and epidemic contributions. More specifically, we assume a conditional intensity function of the form

$$
f(t)=\lambda+\sum_{i} b \exp \left(-a\left(t-t_{i}\right)\right) \text {. }
$$

The above form is motivated by the following interpretation. The terms on the RHS could be interpreted as follows: the intensity function $f(t)$ which consists of two parts:

(1) The external rate of infection which occurs at a constant rate $\lambda$.

(2) The contagion rate due to the already infected persons consists of a constant jump $b$ at the time of infection which decays at a constant rate $a$ so that the contribution to the effective contagion rate at later time $t$ due to an infection at time $t_{i}$ is $b \exp \left(-a\left(t-t_{i}\right)\right)$. Thus the net contribution of the infection rate at time $t$ due to all previous infections at $t_{1}, t_{2}, \ldots, t_{n}$ is

$$
\sum_{i} b \exp \left(-a\left(t-t_{i}\right)\right) \text {. }
$$

The rate could as well be interpreted as a rate at which the instantaneous jump $b$ decreases due to intervention action.

Let $\pi(y, t) d y$ be the probability that $f(t)$ has a value between $y$ and $y+d y$ at time $t$. Writing the forward equation,

$$
\begin{aligned}
\pi(y, t+\Delta) d y= & \pi(y-b ; t)(y-b) \Delta d y \\
& +(1-y \Delta) \pi(y-a(\lambda-y) \Delta ; t) \\
& +o(\Delta) .
\end{aligned}
$$

The above equation is obtained by noting that the rate of decrease of the infection rate is specified by

$$
\frac{d y}{d t}=a(\lambda-y)
$$

Thus (18) reduces to

$$
\begin{aligned}
\frac{\partial \pi}{\partial t}= & (y-b) \pi(y-b ; t)+(a-y) \pi(y, t) \\
& -a(\lambda-y) \frac{\partial \pi}{\partial y} .
\end{aligned}
$$

Letting

$$
P(n, t)=\int_{0}^{\infty} y^{n} \pi(y, t) d y
$$

(20) reduces to

$$
\begin{aligned}
\frac{\partial P(n, t)}{\partial t}= & \operatorname{an\lambda } P(n-1, t)-\operatorname{an} P(n, t) \\
& +\sum_{i}\left(\begin{array}{l}
n \\
i
\end{array}\right) b^{i} P(n-i+1, t)
\end{aligned}
$$


with initial condition

$$
\begin{aligned}
& P(0,1)=1, \\
& P(n, 0)=\lambda^{n} .
\end{aligned}
$$

Letting $n=1$ and solving (22), we obtain

$$
P(1, t)=\frac{a \lambda}{a-b}-\frac{b \lambda}{a-b} \exp (-(a-b) t) .
$$

When $b=0$, so that the contribution of the second term of the contagion rate is not there and only external factor is present, we obtain $P(1, t)=\lambda$ and $E(N(t))=\lambda t$ which is consistent with the assumptions of the model.

From the solution $P(1, t)$, we are in a position to obtain the expected number of infected persons up to time $t$. To see this we note that $\gamma_{1}(t)$, the product density of order one of infections (i.e., probability an infection occurs between $t$ and $t+\Delta)$, is straight away given by

$$
\gamma_{1}(t)=P(1, t)=\int_{0}^{\infty} y \pi(y, t) d y .
$$

Thus the expected number of infections in $(0, t)$ is given by

$$
\begin{aligned}
E(N(t)) & =\int_{0}^{t} \gamma_{1}(u) d u \\
& =\frac{1}{a-b}\left\{a \lambda t-\frac{b \lambda}{a-b}\left(1-e^{-(a-b) t}\right)\right\} .
\end{aligned}
$$

In a similar form, writing the equation satisfied by the conditional density function $\Pi\left(y_{2}, t_{2} \mid y_{1}, t_{1}, \lambda\right)$ representing the probability that $f(t)$ has a value between $y_{2}$ and $y_{2}+d y_{2}$ at time $t_{2}$ given that it has a value between $y_{1}$ and $y_{1}+d y_{1}$ at time $t_{1}$ and initially $\lambda$, we obtain the second order product density $\gamma_{2}\left(t_{1}, t_{2}\right)$. Then using the relation

$$
E(N(t))^{2}=\int_{0}^{t} \gamma_{1}\left(t_{1}\right) d t_{1}+2 \int_{0}^{t} \int_{0}^{t} \gamma_{2}\left(t_{1}, t_{2}\right) d t_{1} d t_{2}
$$

we obtain explicit expression for the above equation after some calculations, which we do not give as they are lengthy and unwieldy.

\section{Discussion}

We have proposed two stochastic models to analyse the dynamics of the spread of a disease. The core of the models is the force of the infection. Each of the infected individual contributes a nonstationary term to the total force of the infection. This comprises a discrete jump at the instant of infection which could be deterministic or random and a response function. The statistical characteristics of the number of infected persons are obtained from the conditional intensity function. The developed models subsume some interesting models available in the literature.

In the epidemic model, letting $b=0$ in (16) so that the contribution to the intensity function is only from external source at a rate $\lambda$, we note that the mean and variance of the number of infected persons are equal to $\lambda t$ showing that the infection grows according to Poisson process with rate $\lambda$. On the other hand letting $a=0$ so that the contribution to the intensity function from the external source and infected individuals are constants $\lambda$ and $b$, respectively, we are in the domain of a simple stochastic epidemic $[13,14]$. In such a scenario, our model predicts

$$
E(N(t))=\frac{1}{b}\left\{\lambda\left(e^{b t}-1\right)\right\}
$$

and Gielen [14] shows the number of infected persons has a negative binomial distribution with the same mean. In this case with probability 1 , there are ultimately infinitely many infective individuals. Also the mean number of infective individuals obtained using our model satisfies the renewal equation (2) of Keyfitz's deterministic model [15].

With the usual notations, the equations of the susceptibleinfected-recovered (SIR) model are given by

$$
\begin{aligned}
& \frac{d S}{d t}=-\frac{\beta I S}{N}, \\
& \frac{d I}{d t}=\frac{\beta I S}{N}-\vartheta I, \\
& \frac{d R}{d t}=\vartheta I .
\end{aligned}
$$

These equations assume that infected people cause infections at rate $\beta$ and recover at rate $\vartheta$, giving an exponentially distributed infection duration. The process is obviously memoryless. In contrast, in real life situations, for diseases the "age" of an individual's infection affects his/her infectiousness and consequently probability of recovery. Thus our modeling could be visualised as transcending the memoryless regime.

Gielen [14], in his model, considered the intensity function of the form

$$
f(t)=a(t)+\sum g\left(t-t_{i}\right) .
$$

In such case, the total contribution to the infectivity function by the external source and by an infective during the entire infective period, respectively, is given by

$$
\begin{aligned}
& \int_{0}^{\infty} f(t) d t=F(\infty), \\
& \int_{0}^{\infty} g(t) d t=G(\infty) .
\end{aligned}
$$

These two quantities are dimensionless. If $F(\infty)=\infty$, then, with probability 1 , there are finally infinitely many defectives which is the case in our model (see page 8 of the paper, [14]). However in Gielen's model, it is possible that $0<F(\infty)=$ : $A<\infty$ and $G(\infty)=\infty$. In such a scenario, there is a nonzero probability that infection may not start at all so that the probability of infinite infective individuals ultimately is defective with probability $1-e^{-A}$. In our model we start with $f(0)=\lambda$ which preclude such an eventuality.

In our endemic model, letting $a=1$ and $h(t)=\delta(t-$ 1) where $\delta$ is the Dirac delta function, we note that each 
infection increases the force of the infectivity by one unit. This is reflected in the final solution as we get

$$
\Pi(n, t)=P(f(t)=n)=\frac{e^{-\lambda t}(\lambda t)^{n}}{n !}, \quad n=0,1, \ldots
$$

Thus we arrive at a Poisson process of infections. In the endemic model, the contribution to the force of infectivity arises only from the external source. If we further assume that infections also occur due to the already infected persons but such infected individuals are removed from the pool of infected, then the probability of an infection due to the infected population in $(t, t+d t]$ is given by

$$
\int_{0}^{\infty} y \Pi(y, t) d y d t
$$

This can be identified with the product density of order one, namely, $\gamma_{1}(t)$. Hence the expected number of infected individuals at time $t$ is

$$
\int_{0}^{t} \gamma_{1}(u) d u
$$

while the expected number of infected persons due to external source is $\lambda t$.

Finally, we wish to mention the useful tools that a planner needs in designing interventions is the estimated values of the model parameters. In order to estimate the parameters of our model, $a$ and $b$, we need the function

$$
\gamma_{n}\left(t_{1}, t_{2}, \ldots, t_{n} ; a, b\right)
$$

which is the product density of order $n$. This function which can serve as the likelihood function specifies the probability that infections occur at $t_{1}, t_{2}, \ldots, t_{n}$ under the assumed values of $a$ and $b$. Although such a function could be obtained, it may not be of much value in actual use due to its unwieldy form. Thus we make the reasonable approximation

$$
\begin{aligned}
& \gamma_{n}\left(t_{1}, t_{2}, \ldots, t_{n} ; a, b\right) \\
& \quad=\gamma_{1}\left(t_{1} ; a, b\right) \gamma_{1}\left(t_{2}-t_{1} ; a, b\right) \cdots \gamma_{1}\left(t_{n}-t_{n-1} ; a, b\right) .
\end{aligned}
$$

The estimate of the parameters $a$ and $b$ yields the maximum likelihood parameters $\widehat{a}$ and $\widehat{b}$, by using methods proposed by Rubin [18] and Ozaki [19].

This paper generalizes the stochastic version of Kermack and McKendrick model of Gielen [14] by considering the transient behaviour of the total infectivity present in an infinite population of susceptible individuals. A noteworthy aspect is the explicit analytical expressions for the probability distribution and statistical characteristics of the number of infected individuals. The proposed models can also be solved using Lie algebra approach for finding analytical solutions. The proposed model could be useful in order to clarify theoretical concepts on infectiousness, the force of the infection, in order to compare the infectiousness of different communicable diseases such as Ebola virus disease. The future work is proposed to carry out specific statistical analysis and estimation of parameters using real-life data for epidemic disease.

\section{Competing Interests}

The authors declare that they have no competing interests.

\section{Acknowledgments}

The authors thank Professor Alagar Rangan for constructive comments and considerable support on this project during his visit to Universidad Nacional de Colombia.

\section{References}

[1] W. O. Kermack and A. G. McKendrick, "A contribution to the mathematical theory of epidemics," Proceedings of the Royal Society of London Series A: Mathematical, Physical and Engineering Sciences, vol. 115, no. 772, pp. 700-721, 1927.

[2] S. Meyer, J. Elias, and M. Höhle, "A space-time conditional intensity model for invasive meningococcal disease occurrence," Biometrics. Journal of the International Biometric Society, vol. 68, no. 2, pp. 607-616, 2012.

[3] A. Dassios and H. Zhao, "A dynamic contagion process," Advances in Applied Probability, vol. 43, no. 3, pp. 814-846, 2011.

[4] G. Streftaris and G. J. Gibson, "Bayesian inference for stochastic epidemics in closed populations," Statistical Modelling, vol. 4, no. 1, pp. 63-75, 2004.

[5] L. Tierney, "Markov chains for exploring posterior distributions," The Annals of Statistics, vol. 22, no. 4, pp. 1701-1762, 1994.

[6] V. Andreasen, "Instability in an SIR-model with age-dependent susceptibility," in Mathematical Population Dynamics: Analysis of Heterogeneity Volume 1: Theory of Epidemics, O. Arino, D. Axelrod, M. Kimmel, and M. Langlais, Eds., p. 314, Wuerz Publishing, Winnipeg, Canada, 1995.

[7] T. Britton, "Stochastic epidemic models: a survey," Mathematical Biosciences, vol. 225, no. 1, pp. 24-35, 2010.

[8] H. C. Tuckwell and R. J. Williams, "Some properties of a simple stochastic epidemic model of SIR type," Mathematical Biosciences, vol. 208, no. 1, pp. 76-97, 2007.

[9] Y. Shang, "Mixed SI (R) epidemic dynamics in random graphs with general degree distributions," Applied Mathematics and Computation, vol. 219, no. 10, pp. 5042-5048, 2013.

[10] T. House, "Lie algebra solution of population models based on time-inhomogeneous Markov chains," Journal of Applied Probability, vol. 49, no. 2, pp. 472-481, 2012.

[11] Y. Shang, "A lie algebra approach to susceptible-infectedsusceptible epidemics," Electronic Journal of Differential Equations, vol. 2012, article 233, pp. 1-7, 2012.

[12] J. L. W. Gielen, "Epidemics between Bailey and reed-frost: a stochastic version of the Kermack and McKendrick model," Journal of Biological Systems, vol. 5, no. 4, pp. 489-508, 1997.

[13] N. T. J. Bailey, The Mathematical Theory of Infectious Diseases and Its Applications, The Griffin, London, UK, 2nd edition, 1975.

[14] J. L. W. Gielen, "A stochastic model for epidemics based on the renewal equation," Journal of Biological Systems, vol. 8, no. 1, pp. $1-20,2000$.

[15] N. Keyfitz, Introduction to the Mathematics of Population, Addison-Wesley, Reading, Mass, USA, 1968.

[16] G. Chowell and H. Nishiura, "Transmission dynamics and control of Ebola virus disease (EVD): a review," BMC Medicine, vol. 12, article 196, 2014. 
[17] H. Nishiura and G. Chowell, "Early transmission dynamics of Ebola virus disease (evd), West Africa, march to august 2014," Eurosurveillance, vol. 19, no. 36, pp. 1-6, 2014.

[18] I. Rubin, "Regular point processes and their detection," IEEE Transactions on Information Theory, vol. 18, pp. 547-557, 1972.

[19] T. Ozaki, "Maximum likelihood estimation of Hawkes' selfexciting point processes," Annals of the Institute of Statistical Mathematics, vol. 31, no. 1, pp. 145-155, 1979. 


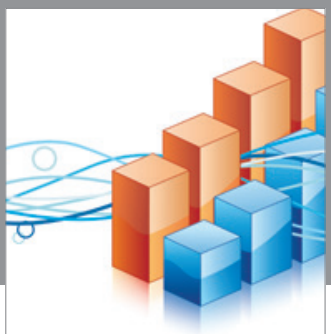

Advances in

Operations Research

vatem alat4

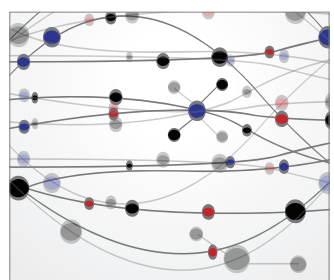

\section{The Scientific} World Journal
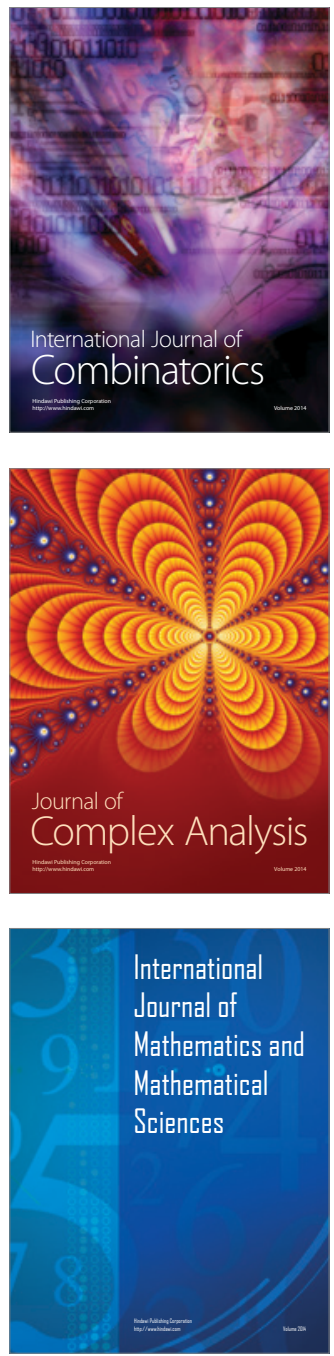
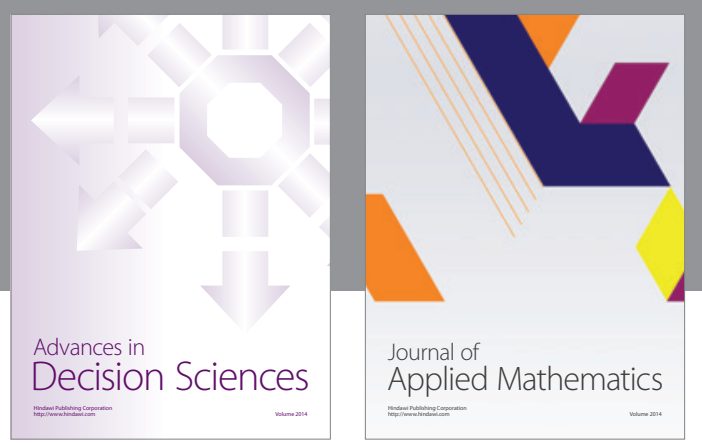

Algebra

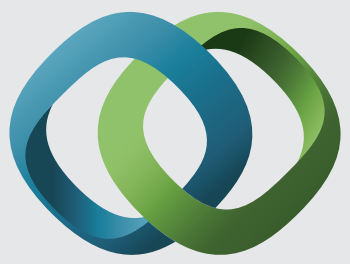

\section{Hindawi}

Submit your manuscripts at

https://www.hindawi.com
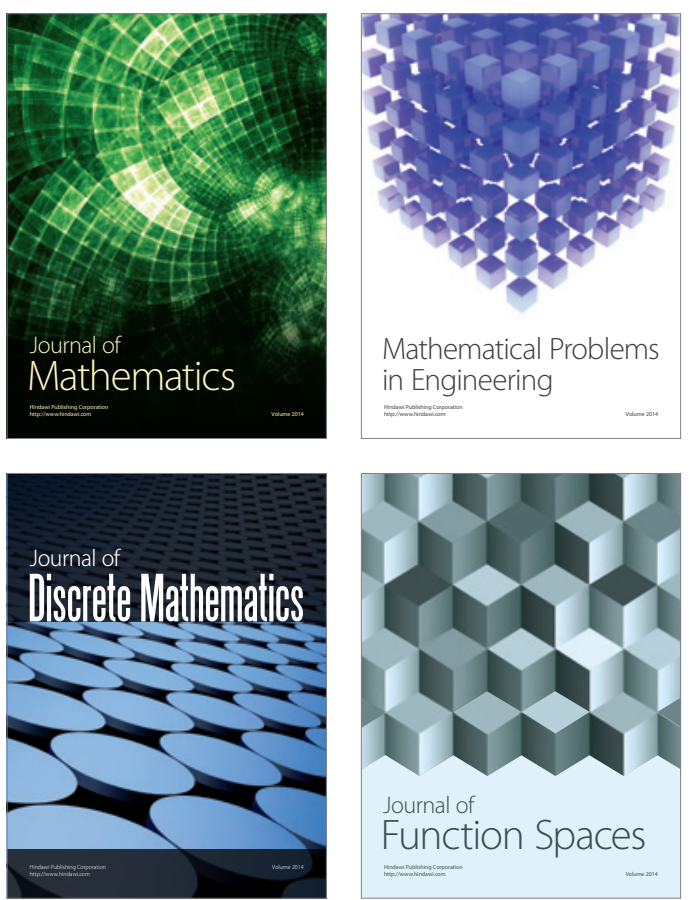

Mathematical Problems in Engineering
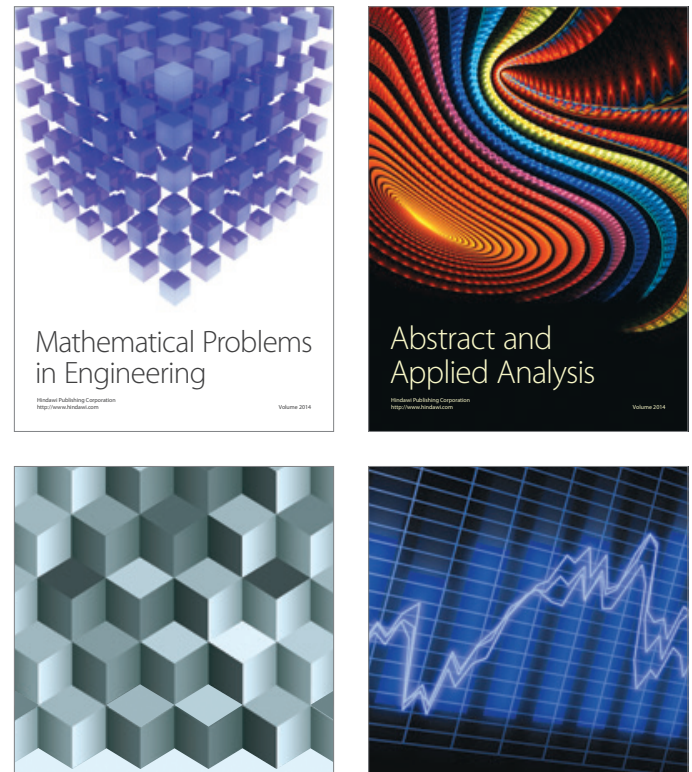

Journal of

Function Spaces

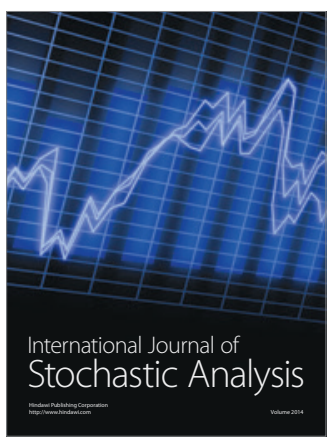

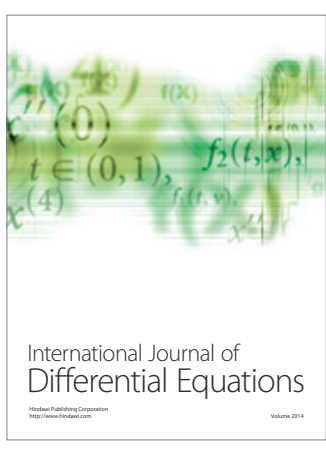
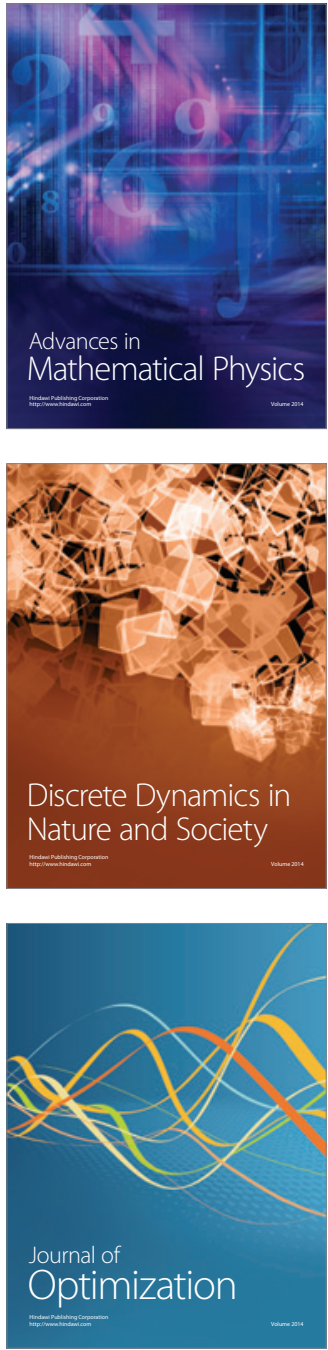